\title{
Indicadores socioeconómicos y prevalencia de anemia- menores de 3 años, Centro Salud Sapallanga, 2017
}

\author{
Socioeconomic indicators and prevalence of anemia - children under 3 years, Sapallanga Health Center, \\ 2017
}

\author{
Belinda Olga García Inga 1,a, Jenny Giovana Poma Salinas 1,b, Edith Suasnabar Cueva ${ }^{1, b}$, \\ Rebeca Tello Carhuanca ${ }^{1, a}$
}

\section{RESUMEN}

Objetivos: La situación de la anemia en el Centro de Salud de Sapallanga se encuentra elevada y alcanza una tasa de prevalencia de $57 \%$ del total de niños que cuenta dicho distrito lo que es preocupante y fue el motivo de esta investigación, habiéndonos formulado como problema principal: ¿Qué indicadores socioeconómicos están relacionados con la prevalencia de anemia en los niños menores de 3 años atendidos en el Centro de Salud de Sapallanga en el 2017? Material y Métodos: Fue de tipo básico, nivel correlacional y de corte transversal. La población y a la vez muestra; del presente estudio comprendió un total de 70 niños menores de 3 años con anemia que se tiene en el Centro de Salud de Sapallanga según padrón nominal. Conclusiones: Al término de la investigación se ha hallado que existen indicadores socioeconómicos que están relacionados significativamente con la prevalencia de anemia en los niños menores de 3 años atendidos en el Centro de Salud de Sapallanga en el 2017 y son: servicio de desagüe en red pública ( $\mathrm{p}$ valor $=0,028<0,05)$; servicio de electricidad ( $\mathrm{p}$ valor $=0,000<0,05)$; trabajo del padre $(\mathrm{p}$ valor $=0,016<0,05)$ y número de integrantes por habitación $(\mathrm{p}$ valor $=0,000<0,05)$.

PALABRAS CLAVE: Indicadores socio económicos, anemia

\section{SUMMARY}

Objectives: The situation of anemia in the Sapallanga Health Center is high and reaches a rate of The prevalence of $57 \%$ of the total number of children in this district is worrisome and was the reason for this research, having formulated us as the main problem: What socioeconomic indicators are related to the prevalence of anemia in children under 3 years of age served in the health center of Sapallanga in 2017? Material and Methods: Was basic, correlated and cross-sectional level. The population and at the same time shows; of the present study comprised a total of 70 children under 3 years of age with anemia in the Sapallanga Health Center according to the nominal standard. Conclusions: At the end of the investigation, it was found that there are socioeconomic indicators that are significantly related to the prevalence of anemia in children under 3 years of age attended in the health center of Sapallanga in 2017 and are: Public network drain service ( $p$ value $=0.028<0.05$ ); electricity service ( $p$ value $=0.000<0.05$ ); father's work ( $\mathrm{p}$ value $=0.016<0.05)$ and number of members per room ( $\mathrm{p}$ value $=0.000<0.05)$.

KEYWORDS: Socioeconomic indicators, anemia 


\section{INTRODUCCIÓN}

A nivel internacional la anemia representa un problema grande de salud pública por su alta prevalencia y por presentarse especialmente en niños y mujeres en edad fértil. En cifras absolutas, estos porcentajes representan 293 millones de niños menores de 5 años, y 468 millones de mujeres no embarazadas afectados por anemia en el mundo. En Latinoamérica, la prevalencia de anemia en niños menores de cinco años es del $29,3 \%$, lo cual corresponde aproximadamente 23 millones de niños afectados. Asimismo, la prevalencia en mujeres en edad fértil en Latinoamérica es de 17,8\% (39 millones de afectadas).

En el Perú, la anemia en los niños menores de 5 años es muy prevalente, aunque algunos avances se han hecho respecto a su control en las últimas dos décadas. Según la Encuesta Nacional Demográfica de Salud (ENDES) del Ministerio de Desarrollo e Inclusión Social (MIDIS), que es un estudio poblacional, de representatividad nacional y regional, los indicadores socioeconómicos que se utilizan en nuestro país para evaluar los avances de la disminución de la pobreza, el analfabetismo y otros determinantes sociales negativos que influyen en la salud de la población; de allí la importancia de mencionar al Ministerio de Desarrollo e Inclusión Social (MIDIS), que es un organismo del Poder Ejecutivo de nuestro país (1).

El propósito de esta investigación fue contribuir con la disminución de los indicadores negativos en salud como la anemia en nuestra población, general y por ende en el distrito de Sapallanga. El estudio contiene el planteamiento del problema donde se formulan los problemas y se plantean los objetivos de investigación. Contiene también el marco teórico donde se mencionan los antecedentes referentes al estudio tanto internacionales como nacionales, la base teórica, el marco conceptual, las hipótesis y las variables de estudio. Se da a conocer también la metodología que se usó para este estudio, así como los resultados, la discusión de los mismos para llegar a las conclusiones de la investigación.

\section{MATERIAL Y MÉTODOS}

En el presente estudio se hará uso del método científico; Según Ruiz: es el procedimiento planteado que se sigue en la investigación para descubrir las formas de existencia de los procesos objetivos, para desentrañar sus conexiones internas y externas, para generalizar y profundizar los conocimientos así adquiridos, para llegar a demostrarlos con rigor racional y para comprobarlos en el experimento y con las técnicas de su aplicación (2).

El tipo de investigación del presente estudio es aplicada. Regalado Bernal sostiene que este tipo de investigación, nos lleva a la búsqueda del conocimiento de la causa del fenómeno investigado (3).

El nivel de investigación del presente estudio es correlacional, porque consiste en medir el grado de relación o asociación que existe entre dos o más variables.

El diseño de la investigación es transeccionalcorrelacional causal que son aquellos que describen efectos entre dos o más categorías, conceptos o variables en un momento determinado, donde las causas y los efectos ya ocurrieron en la realidad (estaban dados y manifestados) o suceden durante el desarrollo del estudio, y quien investiga los observa y reporta, cuyo esquema es:

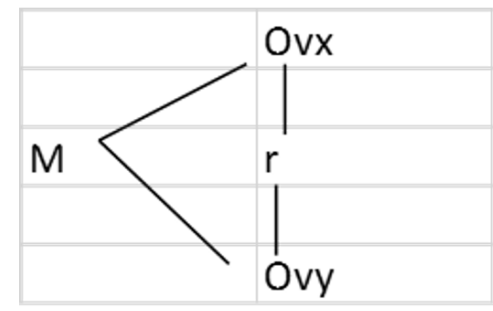

Donde:

$\mathrm{M}=$ representa la muestra de estudio.

$\mathrm{O}=$ representa la información relevante de la muestra u objeto de estudio.

$\mathrm{r}=$ representa a la posible relación existente entre las variables de estudio

La población del presente estudio comprende un total de 70 niños menores de 3 años con anemia del Centro de Salud de Sapallanga según padrón nominal. En el presente estudio de investigación no se aplicó muestra probabilística, porque se va trabajar con toda la población de estudio, que comprende un total de 70 niños menores de 3 años con anemia.

Sin embargo, el tipo de muestreo del estudio es no probabilístico de tipo intencional con dos grupos de estudio en el mismo período de estudio.

El grupo casos fue conformado por los 70 niños menores de 03 años con anemia. 
El grupo control fue conformado por 140 niños menores de 3 años sin anemia.

La técnica de investigación que se utilizó en el presente estudio para la recolección de datos de la variable uno, es la técnica documental: que consiste en recopilar datos de las historias clínicas, como conocimientos, ideas y opiniones de grupos; aspectos que se analizan con el propósito de determinar rasgos de las personas, proponer o establecer relaciones entre las características de los sujetos, lugares y situaciones o hechos.28 El instrumento fue la ficha de recolección de datos.

El instrumento para la recolección de datos de la variable dos; en el presente estudio será la ficha de recolección de datos, con la cual se recolectará información de los niños con anemia.29

\section{Confiabilidad}

Prueba Piloto: 30, Ítems: 10

Estadisticas de fiabilidad

\begin{tabular}{|c|c|}
\hline Alfa de & Número de \\
\hline Cronbach & elementos \\
\hline 0,804 & 10 \\
\hline
\end{tabular}

De acuerdo a Rosas y Zúñiga, el instrumento es confiable para una investigación explicativa a partir de 0,8 (4), por lo tanto, el valor de Alfa de Cronbach obtenido de 0,804 nos indica que el instrumento de esta investigación es confiable.

\section{RESULTADOS}

Los resultados se hallaron a partir de la base de datos SPSS V23, obteniendo resultados descriptivos e inferenciales.

\section{Resultados descriptivos}

Se presentan en dos partes: Resultados de indicadores sociales $\mathrm{y}$ resultados de indicadores económicos.

\section{Resultados del indicador identidad}

La tabla 1 nos muestra los resultados en porcentajes y frecuencias acerca del Registro del Niño en RENIEC, es así que en los resultados de casos el 97,8\% respondió Sí 2,2\% No. Siendo similares los resultados de Control, donde el 97,8\% respondió Sí $2,2 \%$ No.

\section{Resultado del indicador Grado de Instrucción}

a) De instrucción de la madre

La tabla 2 nos muestran los resultados en porcentajes y frecuencias acerca del Grado de instrucción de la madre, es así que en los resultados de Casos, el mayor

Tabla 1. Registro del Niño en RENIEC

\begin{tabular}{lccccc}
\hline & \multicolumn{2}{c}{ CASOS } & & \multicolumn{2}{c}{ CONTROL } \\
\cline { 2 - 3 } \cline { 5 - 6 } & Frecuencia & Porcentaje & & Frecuencia & Porcentaje \\
\hline SI & 88 & $97.8 \%$ & & 176 & $97.8 \%$ \\
NO & 2 & $2.2 \%$ & & 4 & $2.2 \%$ \\
Total & 90 & $100.0 \%$ & & 180 & $100.0 \%$ \\
\hline Fuente: Ficha de Recolección de datos & & & &
\end{tabular}

Tabla 2. Grado de instrucción de la madre

\begin{tabular}{lccccc}
\hline & \multicolumn{2}{c}{ CASOS } & & \multicolumn{2}{c}{ CONTROL } \\
\cline { 2 - 3 } \cline { 5 - 6 } & Frecuencia & Porcentaje & & Frecuencia & Porcentaje \\
\hline INICIAL & 1 & $1.1 \%$ & & 2 & $1.1 \%$ \\
PRIMARIA & 16 & $17.8 \%$ & & 22 & $12.2 \%$ \\
SECUNDARIA & 58 & $64.4 \%$ & & 114 & $63.3 \%$ \\
SUPERIOR & 15 & 16.7 & & 42 & $23.3 \%$ \\
SIN INSTRUCCIÓN & 0 & $0.0 \%$ & & 0 & $0.0 \%$ \\
Total & 90 & $100.0 \%$ & & 180 & $100.0 \%$ \\
\hline Fuente: Ficha de Recolección de datos & & & &
\end{tabular}


porcentaje $64,4 \%$ estudiaron hasta secundaria; $1,1 \%$ inicial, $17,8 \%$ primaria, $16,7 \%$ superior y el $0 \% \sin$ instrucción.

En tanto que los resultados de Control, el mayor porcentaje $63,3 \%$ estudiaron hasta secundaria; $1,1 \%$ inicial, $12,2 \%$ primaria, $23,3 \%$ superior y el $0 \% \sin$ instrucción.

b) Grado de instrucción del padre

La tabla 3 nos muestra los resultados en porcentajes y frecuencias acerca del Grado de instrucción del padre, es así que en los resultados de Casos el mayor porcentaje $77,8 \%$ estudiaron hasta secundaria; $0 \%$ inicial, $13,3 \%$ primaria, $8,9 \%$ superior y el $0 \% \sin$ instrucción.

En tanto que los resultados de Control, el mayor porcentaje $72,2 \%$ estudiaron hasta secundaria; $0 \%$ inicial, $11,1 \%$ primaria, $16,7 \%$ superior y el $0 \% \sin$ instrucción.

\section{Resultados del indicador acceso a servicios básicos}

a) Servicio de agua potable

La tabla 4 nos muestra los resultados en porcentajes y frecuencias acerca del servicio de agua potable, es así que, en los resultados de Casos, sí cuentan con este servicio el $94,4 \%$ y no $5,6 \%$. En tanto que los resultados de Control, sí cuentan con este servicio el $96,7 \%$ y no $3,3 \%$.

b) Servicio de desagüe

La tabla 5 nos muestra los resultados en porcentajes y frecuencias acerca del servicio de desagüe, es así que, en los resultados de Casos, sí cuentan con este servicio el $57,8 \%$ y no $42,2 \%$. En tanto que los resultados de Control, sí cuentan con este servicio el $71,1 \%$ y no $28,9 \%$.

c) Servicio de electricidad

La tabla 6 nos muestra los resultados en porcentajes y frecuencias acerca del servicio de electricidad, es así que, en los resultados de Casos, sí cuentan con este servicio el $94,4 \%$ y no $5,6 \%$. En tanto que los resultados de Control, sí cuentan con este servicio el $96,7 \%$ y no $3,3 \%$.

\section{Resultados del indicador vivienda}

La tabla 7 nos muestra los resultados en porcentajes y frecuencias acerca del material de construcción de la vivienda, es así que en los resultados de Casos el $37,8 \%$ respondió material noble y $62,2 \%$ material

Tabla 3. Grado de instrucción de la padre

\begin{tabular}{lccccc}
\hline & \multicolumn{2}{c}{ CASOS } & & \multicolumn{2}{c}{ CONTROL } \\
\cline { 2 - 3 } \cline { 5 - 6 } \cline { 5 - 6 } & Frecuencia & Porcentaje & & Frecuencia & Porcentaje \\
\hline INICIAL & 0 & $0.0 \%$ & & 0 & $0.0 \%$ \\
PRIMARIA & 12 & $13.3 \%$ & & 20 & $11.1 \%$ \\
SECUNDARIA & 70 & $77.8 \%$ & & 130 & $72.2 \%$ \\
SUPERIOR & 8 & $8.9 \%$ & & 30 & $16.7 \%$ \\
SIN INSTRUCCIÓN & 0 & $0.0 \%$ & & 0 & $0.0 \%$ \\
Total & 90 & $100.0 \%$ & & 180 & $100.0 \%$ \\
\hline Funnnyyyn & & & & &
\end{tabular}

Tabla 4. Servicio De Agua Potable

\begin{tabular}{lccccc}
\hline & \multicolumn{2}{c}{ CASOS } & & \multicolumn{2}{c}{ CONTROL } \\
\cline { 2 - 3 } \cline { 5 - 6 } \cline { 5 - 6 } & Frecuencia & Porcentaje & & Frecuencia & Porcentaje \\
\hline SI & 85 & $94.4 \%$ & & 174 & $96.7 \%$ \\
NO & 5 & $5.6 \%$ & & 6 & $3.3 \%$ \\
Total & 90 & $100.0 \%$ & & 180 & $100.0 \%$ \\
\hline
\end{tabular}

Fuente: Ficha de Recolección de datos 
Tabla 5. Servicio de desagüe

\begin{tabular}{llllll}
\hline & \multicolumn{2}{c}{ Casos } & & \multicolumn{2}{c}{ Control } \\
\cline { 2 - 3 } \cline { 5 - 6 } \cline { 5 - 6 } & Frecuencia & Porcentaje & & Frecuencia & Porcentaje \\
\hline SI & 52 & $57,8 \%$ & & 128 & $71,1 \%$ \\
NO & 38 & $42,2 \%$ & & 52 & $28,9 \%$ \\
Total & 90 & $100,0 \%$ & & 180 & $100,0 \%$ \\
\hline
\end{tabular}

Fuente: Ficha de Recolección de datos

Tabla 6. Servicio de electricidad

\begin{tabular}{lcccc}
\hline & Frecuencia & Porcentaje & Frecuencia & Porcentaje \\
\cline { 2 - 5 } SI & 85 & $94,4 \%$ & 174 & $96,7 \%$ \\
NO & 5 & $5,6 \%$ & 6 & $3,3 \%$ \\
Total & 90 & $100,0 \%$ & 180 & $100,0 \%$ \\
\hline
\end{tabular}

Tabla 7. Material de construcción de la vivienda

\begin{tabular}{lccccc}
\hline & \multicolumn{2}{c}{ Casos } & & \multicolumn{2}{c}{ Control } \\
\cline { 2 - 3 } \cline { 5 - 6 } \cline { 5 - 6 } & Frecuencia & Porcentaje & & Frecuencia & Porcentaje \\
\hline MATERIAL NOBLE & 34 & $37,8 \%$ & & 96 & $53,3 \%$ \\
MATERIAL RÚSTICO & 58 & $62,2 \%$ & & 84 & $46,7 \%$ \\
Total & 90 & $100,0 \%$ & & 180 & $100,0 \%$ \\
\hline
\end{tabular}

rústico. En tanto que los resultados de Control, el $53,3 \%$ respondió material noble y $46,7 \%$ material rústico.

\section{Resultados del indicador trabajo}

\section{a) Ocupación de la madre}

La tabla 8 nos muestra los resultados en porcentajes y frecuencias acerca de la ocupación de la madre, es así que en los resultados de Casos el mayor porcentaje $62,2 \%$ son trabajadoras del hogar, $2,2 \%$ obreras, $17,8 \%$ trabajadoras independientes, y $17,8 \%$ trabajador familiar no remunerado. En tanto que los resultados de Control, el mayor porcentaje $55,6 \%$ son trabajadoras del hogar, $6,7 \%$ obreras, $0 \%$ trabajadoras independientes, y $15,6 \%$ trabajador familiar no remunerado.

\section{b) Ocupación del padre}

La tabla 9 nos muestra los resultados en porcentajes y frecuencias acerca de la ocupación del padre, es así que en los resultados de Casos el mayor porcentaje
$81,1 \%$ son trabajadores independientes, $4,4 \%$ empleados, $7,8 \%$ obreros, $0 \%$ trabajadores del hogar $17,8 \%$, y $6,7 \%$ trabajador familiar no remunerado.

En tanto que los resultados de Control, el mayor porcentaje $68,9 \%$ son trabajadores independientes, $8,9 \%$ empleados, $16,7 \%$ obreros, $0 \%$ trabajadores del hogar $17,8 \%$, y $5,6 \%$ trabajador familiar no remunerado.

\section{Resultados del indicador número de miembros}

La tabla 10 nos muestra los resultados en porcentajes y frecuencias acerca del número de integrantes por habitación, es así que en los resultados de Casos donde la mayoría $56,7 \%$ respondió tres a más miembros por habitación, 2,2\% un miembro por habitación y 41,1\% dos miembros por habitación.

En tanto que los resultados de Control, la mayoría $38,9 \%$ respondió tres a más miembros por habitación, $26,7 \%$ un miembro por habitación y $34,4 \%$ dos miembros por habitación. 
Tabla 8. Ocupación de la madre

\begin{tabular}{lccccc}
\hline & \multicolumn{2}{c}{ Casos } & & \multicolumn{2}{c}{ Control } \\
\cline { 2 - 3 } \cline { 5 - 6 } & Frecuencia & Porcentaje & & Frecuencia & Porcentaje \\
\hline EMPLEADO & 2 & $2,2 \%$ & & 12 & $6,7 \%$ \\
OBRERO & 0 & $0,0 \%$ & & 0 & $0,0 \%$ \\
TRABAJADOR INDEPENDIENTE & 16 & $17,8 \%$ & & 40 & $22,2 \%$ \\
TRABAJADOR DEL HOGAR & 56 & $62,2 \%$ & & 100 & $55,6 \%$ \\
TRABAJADOR FAMILIAR NO & 16 & $17,8 \%$ & & 28 & $15,6 \%$ \\
REMUNERADO & 90 & $100,0 \%$ & & 180 & $100,0 \%$ \\
Total & & & & & \\
\hline
\end{tabular}

Tabla 9. Ocupación del padre

\begin{tabular}{lcccccc}
\hline & \multicolumn{2}{c}{ Casos } & & \multicolumn{2}{c}{ Control } \\
\cline { 2 - 3 } \cline { 6 - 7 } & Frecuencia & Porcentaje & & Frecuencia & Porcentaje \\
\hline EMPLEADO & 4 & $4,4 \%$ & & 16 & $8,9 \%$ \\
OBRERO & 7 & $7,8 \%$ & & 30 & $16,7 \%$ \\
TRABAJADOR INDEPENDIENTE & 73 & $81,1 \%$ & & 124 & $68,9 \%$ \\
TRABAJADOR DEL HOGAR & 0 & $0,0 \%$ & & 0 & $0,0 \%$ \\
TRABAJADOR FAMILIAR NO REMUNERADO & 6 & $6,7 \%$ & & 10 & $5,6 \%$ \\
Total & 90 & $100,0 \%$ & & 180 & $100,0 \%$ \\
\hline
\end{tabular}

\section{Prueba de Hipótesis}

\section{Prueba de la Hipótesis General}

Hi: Existen indicadores socioeconómicos que están relacionados significativamente con la prevalencia de anemia en los niños menores de 3 años atendidos en el Centro de Salud de Sapallanga en el 2017.

Ho: No existen indicadores socioeconómicos que están relacionados significativamente con la prevalencia de anemia en los niños menores de 3 años atendidos en el Centro de Salud de Sapallanga en el 2017

Nivel de significancia: $\alpha=0,05$

\section{Conclusión estadística}

Se rechaza la hipótesis nula y se acepta la hipótesis de investigación en el sentido que existen indicadores socioeconómicos que están relacionados significativamente con la prevalencia de anemia en los niños menores de 3 años atendidos en el Centro de Salud de Sapallanga en el 2017 y son: servicio de desagüe en red pública ( $\mathrm{p}$ valor $=0,028<0,05$ ); servicio de electricidad ( $\mathrm{p}$ valor $=0,000<0,05$ ); trabajo del padre ( $\mathrm{p}$ valor $=0,016<0,05)$ y número de integrantes por habitación ( $\mathrm{p}$ valor $=0,000<0,05)$.

\section{DISCUSIÓN}

Los resultados acerca del objetivo general nos muestran que existen indicadores socioeconómicos que están relacionados significativamente con la prevalencia de anemia en los niños menores de 3 años atendidos en el Centro de Salud de Sapallanga en el 2017 y son: servicio de desagüe en red pública ( $\mathrm{p}$ valor $=0,028<0,05)$; servicio de electricidad $(\mathrm{p}$ valor $=$ $0,000<0,05)$; trabajo del padre $(\mathrm{p}$ valor $=0,016<0,05)$ y número de integrantes por habitación ( $\mathrm{p}$ valor $=$ $0,000<0,05)$. 
En este sentido se tienen antecedentes que difieren de esta investigación como la de Velásquez et al., habiendo encontrado que la prevalencia de anemia fue alta $(47,9 \%)$, se identificaron doce factores asociados con la anemia: factores sociodemográficos como vivir fuera de Lima y Callao; en un hogar con bajo nivel socioeconómico; tener una madre adolescente y con bajo nivel educativo; ser de sexo masculino con edad menor de 24 meses y antecedentes de fiebre reciente, y factores relacionados con el cuidado materno-infantil como la falta de control prenatal en el primer trimestre, la falta de suplemento de hierro durante el embarazo o administrado durante un periodo breve, parto en el domicilio, diagnóstico de anemia en la madre en el momento de la encuesta y ausencia de tratamiento antiparasitario preventivo en el niño (5).

Otra investigación que difiere de esta investigación es la de Munares y Gómez, donde el 79,1\% tenían entre 6 a 23 meses, $75,9 \%$ recibieron multimicronutrientes y la adherencia fue del 24,4\% (IC95\% 22,3 - 26,6) (6). Los factores: seguir con la suplementación $(\mathrm{OR}=3,5$; IC95\% 1,7 - 7,5); no tener náuseas ( $\mathrm{OR}=3,0 ; \mathrm{IC} 95 \%$ 2,0 - 4,3); no tomar antibióticos (OR = 2,5; IC95\% 1,7 - 3,6) e intenciones de seguir con el tratamiento $(\mathrm{OR}=$ 2,3; IC95\% 1,3 - 4,1) se asociaron a la adherencia.

El análisis multivariado asoció pensar que debe continuar con el tratamiento (ORa $=2,6$; IC95\% 1,1 $6,1)$; si presentó algún efecto secundario, no suprimió el tratamiento $(\mathrm{ORa}=2,5$; IC95\% 1,4 - 4,3), el niño no tomó antibióticos (ORa $=2,0 ;$ IC95\% 1,1 - 3,4) y creencia que anemia no solo se cura con medicamentos $(\mathrm{ORa}=1,6 ;$ IC95\% 1,0 - 2,6). Conclusión: Hubo una baja prevalencia de adherencia para un punto de corte exigente ( $\geq 90 \%$ sobres de multimicronutrientes consumidos) y los factores asociados están relacionados con ausencia de infecciones, efectos secundarios y creencias de la madre (6).

La teoría acerca de indicadores socioeconómicos que se utilizan en nuestro país para evaluar los avances de la disminución de la pobreza, el analfabetismo y otros determinantes sociales negativos que influyen en la salud de la población, el MIDIS ha establecido una clasificación de los indicadores socioeconómicos con la cual año tras año viene realizando la evaluación del logro de las metas establecidas por cada indicador, siendo estos indicadores socioeconómicos: pobreza, identidad, educación, salud y nutrición (anemia), acceso a servicios básicos, vivienda, trabajo, y otros (1).

\section{CONCLUSIONES}

1) Se ha determinado que existen indicadores socioeconómicos que están relacionados significativamente con la prevalencia de anemia en los niños menores de 3 años atendidos en el Centro de Salud de Sapallanga en el 2017 y son: Servicio de desagüe en red pública ( $\mathrm{p}$ valor $=0,028<0,05$ ); servicio de electricidad ( $\mathrm{p}$ valor $=0,000<0,05$ ); trabajo del padre ( $\mathrm{p}$ valor $=0,016<0,05)$ y número de integrantes por habitación ( $\mathrm{p}$ valor $=$ $0,000<0,05)$.

2) Se ha determinado que no existe relación significativa entre el indicador de identidad y prevalencia de anemia en los niños menores de 3 años atendidos en el Centro de Salud de Sapallanga, 2017. ( $\mathrm{p}$ valor $=1,000>0,05$ ).

3) Se ha determinado que no existe relación significativa entre el indicador de educación y prevalencia de anemia en los niños menores de 3 años atendidos en el Centro de Salud de Sapallanga, 2017. $(\mathrm{p}$ valor $=0,459 ; 0,214>0,05$ ).

4) Se ha determinado que no existe relación significativa entre el indicador de acceso a servicio de agua potable y prevalencia de anemia en los niños menores de 3 años atendidos en el Centro de Salud de Sapallanga, 2017. ( $p$ valor = $0,384>0,05)$, que existe relación significativa entre el servicio de desagüe red pública ( $\mathrm{p}$ valor= $0,028<0,05)$ y que existe relación significativa entre el servicio de electricidad ( $\mathrm{p}$ valor= $0,000<0,05)$

5) Se ha determinado que existe relación significativa entre el indicador de vivienda y prevalencia de anemia en los niños menores de 3 años atendidos en el Centro de Salud de Sapallanga, 2017. (p valor $=0,321>0,05$ ).

6) Se ha determinado que no existe relación significativa entre el indicador trabajo de la madre y prevalencia de anemia en los niños menores de 3 años atendidos en el Centro de Salud de Sapallanga, 2017. ( $\mathrm{p}$ valor $=0,093>0,05$ ). Y que existe relación significativa entre el trabajo del padre y la prevalencia de anemia ( $p$ valor $=$ $0,016<0,05)$

7) Se ha determinado que existe relación significativa entre el indicador número de miembros por hogar y prevalencia de anemia en los niños menores de 3 años atendidos en el Centro de Salud de Sapallanga, 2017. $(\mathrm{p}$ valor $=0,000<0,05)$ 


\section{Correspondencia:}

Belinda Olga García Inga,

Correo electrónico: bgarcia@uroosevelt.edu.pe

\section{REFERENCIAS BIBLIOGRAFICAS}

1. Ministerio de Desarrollo e Inclusión Social. InfoMIDIS. Indicadores socioeconómicos. Lima: Ministerio de Desarrollo e Inclusión Social; 2016. (Citado 20 de setiembre del 2017) Disponible en: http://sdv.midis.gob.pe/Infomidis/\#/ indicadoresSocioEconomicos

2. Ruiz R. El Método Científico y sus etapas. Ciudad de México: CIBERINDEX; 2007.

3. Regalado M. Investigación científica: ¿cómo investigar y preparar tesis de grado? Lima: Compendios Roberman; 1986.
4. Rosas A, Zúñiga J. Estadística Descriptiva e Inferencial. Ciudad de Mexico: Colegio de Bachilleres; 2010.

5. Velásquez JE, Rodríguez $\mathrm{Y}$, Gonzáles $\mathrm{M}$, et al. Factores asociados con la anemia en niños menores de tres años en Perú:análisis de la Encuesta Demográfica y de Salud Familiar, 2007-2013. Biomedica. 2016; 36(2): 0-0.doi: http://dx.doi. org/10.7705/biomedica.v36i2.2896

6. Munares O, Gómez G. Adherencia a multimicronutrientes y factores asociados en niños de 6 a 35 meses de sitios centinela, Ministerio de Salud, Perú. Rev Bras Epidemiol; 19(3): 539-553. doi: http://dx.doi.org/10.1590/1980-5497201600030006.

Recibido: 12/09/2019

Aceptado: 02/11/2019 